\title{
RETRACTED ARTICLE: A distance vector similarity metric for complex networks
}

\author{
Natarajan Meghanathan ${ }^{1}$
}

Received: 28 January 2017 / Accepted: 26 June 2017 / Published online: 4 July 2017

(C) Springer-Verlag GmbH Austria 2017

The editor-in-chief and publisher have retracted this article in agreement with the author. The article was simultaneously submitted to and published online in Computing [DOI 10.1007/s00607-017-0565-6, Natarajan Meghanathan, A distance vector similarity metric for complex networks] and Journal of King Saud University Computer and Information Sciences [DOI 10.1016/j.jksuci.2017.06.007, Natarajan Meghanathan, Decay centrality-based distance vector similarity (DIVES) metric for complex networks]. The online version of this article contains the full text of the retracted article as electronic supplementary material.

Electronic supplementary material The online version of this article (doi:10.1007/s00607-017-0565-6) contains supplementary material, which is available to authorized users.

$凶$ Natarajan Meghanathan

natarajan.meghanathan@jsums.edu

1 Jackson State University, Jackson, MS 39217, USA 